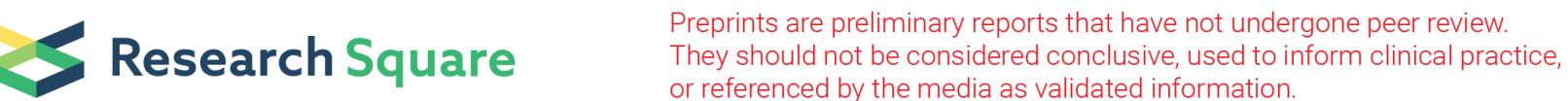

\section{Proportions of Beneficial Factors in MLR, NLR, PLR and D-dimer in Preoperative Peripheral Blood of Patients With Early Stage Lung Cancer as Predictors of Patient Survival After Surgery}

\section{jun wang}

The Second Affiliated Hospital of Harbin Medical University huawei li

The Second Affiliated Hospital of Harbin Medical University ran Xu

The Second Affiliated Hospital of Harbin Medical University tong lu

The Second Affiliated Hospital of Harbin Medical University jiaying zhao

The Second Affiliated Hospital of Harbin Medical University

\section{Pengfei zhang}

The Second Affiliated Hospital of Harbin Medical University

\section{lidong qu}

The Second Affiliated Hospital of Harbin Medical University

\section{Shengqiang zhang}

The Second Affiliated Hospital of Harbin Medical University

\section{Jida guo}

The Second Affiliated Hospital of Harbin Medical University

Linyou zhang ( $\boldsymbol{D}$ lyzhang@hrbmu.edu.cn )

The Second Affiliated Hospital of Harbin Medical University

\section{Research Article}

Keywords: Peripheral blood biomarkers, Early lung cancer, MLR, NLR, PLR, D-dimer

Posted Date: December 30th, 2021

DOI: https://doi.org/10.21203/rs.3.rs-1194999/v1

License: (c) (i) This work is licensed under a Creative Commons Attribution 4.0 International License. 
Page $2 / 18$ 


\section{Abstract}

\section{Objective}

The purpose of this paper is to predict the following items. preoperative baseline monocyte-to-lymphocyte ratio (MLR) $\square$ neutrophil-to-lymphocyte ratio (NLR) Platura-to-lymphocyte ratio (PLR) and dimeric fibrin fragment $D$ (D-dimer) associated with clinical outcome in patients with Early Lung Cancer (LC).

\section{Methods}

We performed a retrospective analysis of 376 patients with LC. Progression-free survival (PFS) and overall survival (OS) were assessed by Kaplan-Meier, and univariate and multivariate Cox regression analyses were performed to identify prognostic factors. Finally, multivariate Cox regression analysis was used to evaluate the influence of favorable factors on patients' OS and PFS combined with the basic clinical characteristics of the patient

Results

Among the variables screened by univariate Cox regression, $\mathrm{MLR}<0.22$, NLR $<1.99$, $\mathrm{PLR}<130.55$ and $\mathrm{D}$ Dimer $<70.5(\mathrm{ng} / \mathrm{ml})$ were significantly associated with both better OS and PFS. In multivariate Cox regression analysis, it was determined that MLR and D-Dimer had a better independent correlation with OS ( $p=0.009, p=0.05$, respectively), while MLR was only better independently associated with PFS $(P=$ 0.005). Furthermore, according to the number of favorable factors, patients with none of these factors had a significantly worse prognosis than patients with at least one of these factors.

Conclusion

Baseline characteristics of low MLR, low NLR, low PLR and low D-dimer were associated with better outcomes.

\section{Background}

With the increasing morbidity and mortality in China, malignant tumors have become the main cause of death, among which lung cancer is the most common cancer and the main cause of death ${ }^{1,2}$. With the development of science and technology, the treatment of lung cancer also presents a variety of methods including surgery, chemotherapy and immunotherapy.However, due to the new coronavirus epidemic, some operations cannot be performed in time. Nevertheless, studies have shown that the benefit of delayed surgical treatment for patients with early-stage non-small cell lung cancer is still better than immediate radiotherapy ${ }^{3}$. Nevertheless, there is still a wide variation in OS and PFS in post-operative patients. Currently, the prediction of survival in postoperative lung cancer patients relies mainly on tumor node metastases (TNM) staging ${ }^{4}$. Although genetic and some molecular tests have shown great promise in predicting patient prognosis ${ }^{5}$, their huge financial burden makes it difficult to be widely available in 
most otherwise affluent cancer families. Therefore, we are working to identify novel circulating biomarkers that can successfully predict patient outcomes during routine preoperative testing.

In recent years, many studies have demonstrated the important role of peripheral blood markers in the prognosis of patients with various tumors. Patients with bladder cancer with a low NLR ${ }^{6}$ had significantly better clinical survival outcomes than those with high NLR ${ }^{6}$. In patients with HER2+ breast cancer, lower NLR and lower MLR show longer survival status ${ }^{6,7}$. It was reported that Plasma D-dimer was regarded as a prognostic marker for various types of malignancies, which included non-small-cell lung carcinoma $(\mathrm{NSCLC})^{8}$. In addition, peripheral blood biomarkers have shown good prognostic ability in a variety of solid tumors including melanoma ${ }^{9}$, colorectal cancer $^{10}$, esophageal cancer ${ }^{11}$ and pancreatic cancer ${ }^{12}$. The above studies strongly suggest that single peripheral blood biomarkers have good prognostic ability in patients with malignancies, but whether there is a superimposed effect or whether these peripheral blood parameter indicators interact with each other needs further elucidation.

\section{Methods}

\section{Patients}

This study retrospectively reviewed the medical records of all LC patients with stage I and stage II that who were treated with standard lobectomy at the Department of Thoracic Surgery, Second Affiliated Hospital of Harbin Medical University, Heilongjiang Province, China, from January 2015 to July 2017. Inclusion criteria: (1) preoperative imaging suggestive of a mass confined to a single lung lobe; (2) no distant metastases; (3) no preoperative adjuvant medication; (4) no hematological malignancies; (5) complete clinical and follow-up information; and (6) survival for at least 30 days postoperatively. Patients were followed up every three months after surgery via outpatient clinics or over the phone, with the last follow-up visit for all patients on June 30,2020. This paper has been approved by the Ethics Committee of the Second Affiliated Hospital of Harbin Medical University. Because the study was retrospective, informed consent from patients was not required. Patient data confidentiality rules are consistent with the Declaration of Helsinki.

\section{Data collection}

Peripheral blood biomarkers including neutrophil count $\left(10^{\wedge} 9 / \mathrm{L}\right)$, monocyte count $\left(10^{\wedge} 9 / \mathrm{L}\right)$, lymphocyte count $\left(10^{\wedge} 9 / \mathrm{L}\right)$, platelet count $\left(10^{\wedge} 9 / \mathrm{L}\right)$ and D-dimer $(\mathrm{ng} / \mathrm{ml})$ were collected from the electronic medical record within 3 days prior to the procedure, as well as the patient's age, gender, BMI, underlying disease history, pathology profile and ECOG at that time scores and other basic clinical information. NLR and MLR were calculated as follows: NLR equals neutrophil count/lymphocyte count; Second, MLR equals monocyte count/lymphocyte count and PLR equals platelet count/lymphocyte count. Survival rates were analyzed by PFS and OS.

\section{Statistical analysis}


According to receiver operating Characteristic (ROC) curve analysis, the critical values of MLR, NLR, PLR and D-dimer can be determined and the highest Yorden index (defined as sensitivity + specificity -1 ) can be used to predict OS. This study used SPSS version 25.0 (IBM Corp., Armonk, NY, USA) for baseline statistics of patient clinical data. Moreover, OS and PFS were analyzed according to the Kaplan-Meier estimate by the log-rank test. Univariate and multivariate Cox regression were used to determine $95 \%$ confidence interval $(\mathrm{Cl})$ and risk ratio $(\mathrm{HR})$. Clinical characterizations, including smoking status (former or never), age, multivariate cox regression was used to analyze the performance of patients in the Eastern tumor cooperative group. A P value less than 0.05 was considered statistically significant. $P<0.05$ on both sides was considered statistically significant.

\section{Results}

\section{Patient demographics and clinical characteristics}

As shown in Table 1, 376 patients with early LC who underwent surgery were finally screened out. The results showed that there were 218 cases $(58 \%)$ in males and 158 cases $(42 \%)$ in females. The median age was $60(53,65)$ years. In our population, the majority of patients had an ECOG physical status of 0-1 $(320 / 376,85.1 \%)$ and the greatest number of patients had lung adenocarcinoma $(n=233 / 376,62 \%)$.

We identified four preoperative peripheral blood biomarkers by referring to relevant studies and combining them with clinical significance ${ }^{13,14}$ Some of the peripheral blood biomarkers for all patients are shown in (Table 2). Basis on the best CUT-OFF, low MLR (239/376, 63,6\%), high NLR (207/376, $55.1 \%)$, low PLR (209/376, 55.6\%) and high D-Dimer (263/376, 69.7\%) were more predominant.

\section{Univariate and Multivariate Cox Analysis for Survival Outcome}

The results of OS and PFS based on cells counts of peripheral blood were shown in Figure 1: pretreatment NLR $<0.216$ (log-rank test $p<0.0001)$, NLR $<1.987(p=0.00016), P L R<130.55(p=0.0034)$ and D-dimer $<70.5 \mathrm{ml} / \mathrm{ng}(0.00016))$. In univariate Cox regressions analysis, sex, smoking, pathology type, MLR, PLR, NLR, D-dimer, and ECOG PS were associated with OS $(p<0.0001,0.0037,0.00021$, 0.00045 and 0.0001 , respectively). The above parameters were correlated with $P F S(P<0.0001,0.01429$, $0.00025,0.01265,0.0001$, respectively) (Table 3 ). In multivariate COX regressions, the preoperative peripheral blood biomarkers MLR and D-Dimer ( $p=0.009145,0.005376$, respectively), which we observed, maintained their significance for OS. However, D-dimer was not significant in PFS ( $p=0.07662)$ (Table 4).

\section{Multivariate Model for Survival of Patients}

Then, according to the quantitative analysis results of favorable factors, including $M L R<0.22, N L R<1.99$, PLR $<130.55$ and D-DIMER $<70.5(\mathrm{ng} / \mathrm{mL}$ ). As shown in Figure 2, Sixty-eight patients (18\%) showed no significant reduction in PFS and OS compared to those who had 1 and 2(group II, at 71 and 93, respectively) or three and four (group III, at 102 and 42, respectively) (Kaplan Meier analysis and survival rates compared to $\mathrm{P}<0.0001$, respectively). Multivariate COX analysis including clinically important 
Covariates (age, gender, BMI, ECOG ps, etc.) confirmed that the number of favorable factors was closely associated to PFS and OS (Table 5).

\section{Discussion}

To date, biomarkers remain a major focus of research in the field of Oncology, whether in the diagnosis of disease, the assessment of efficacy of treatment or the prognosis of patients. The connection between inflammation and cancer was first explored in 1863 by Rudolf Virchow et al. ${ }^{15}$. Since then, more and more studies have further confirmed the value of inflammatory markers in the diagnosis and prognosis evaluation of various malignant tumors. The inflammatory response is an organism's antagonistic response to noxious stimuli, both exogenous and endogenous ${ }^{16,17}$. Inflammation associated with cancer has a dramatic impact on the tumor microenvironment, which consists of tumor cells and inflammatory cells that release various cytokines and chemotactic factors ${ }^{18}$. Among them, NLR, PLR, MLR and D-dimer are closely related to inflammation and immune status of cancer patients, and have been applied to predict the prognosis of patients with various solid tumors ${ }^{9-12}$. NLR, PLR and MLR, as indicators of inflammation, are obtained from peripheral blood neutrophilic granulocyte, platelets and monocytes compared to lymphocytes, respectively. Therefore, the ratios between them can similarly indicate the role of inflammatory mediators in tumors.

The function of inflammatory mediators in the tumor microenvironment is not fully understood, but there are several potential mechanisms that could provide a simple explanation for their role. (1) Lymphocytes are an important component of an organism's immune system, playing a major role in the body's immune surveillance and serving as a protective prognostic factor for patients with malignancies ${ }^{19}$. CD8 cytotoxic T lymphocytes (CTL) are the primary immune cells that target tumors. During cancer progression, CTL become dysfunctional and suppressed due to immune-related tolerance and immunosuppression within the tumor microenvironment $(\mathrm{TME})^{20}$. (2) The role of neutrophilic granulocyte in the body is to regulate immunity by producing tumor necrosis factor (TNF)- $a$, a cytokine that impairs CD8T cell activity and increases vascular permeability, thus suppressing the immune system by inhibiting the immune activity of lymphocytes and ultimately leading to progression and metastasis ${ }^{21,22}$. (3) Monocytes play an important role as a protective immune factor in suppressing tumor growth by inducing the recruitment and function of lymphocytes in TME and interacting with adaptive immunity, especially in peripheral blood where monocytes are involved in paracrine signaling and produce many inflammatory cytokines and chemokines, including tumor necrosis factor $a^{23}$. (4) Platelets in plasma play a vital part in tumor hematogenous metastasis and are a prerequisite for it. The mechanism of action may be that platelets protect tumor cells from shear and NK cell attack ${ }^{24}$. (5) Although the mechanism of plasma D-Dimer in tumor development is still unclear, some studies have reported that elevated plasma D-Dimer levels in breast cancer patients are associated with progesterone receptor expression, TNM staging and metastasis in breast cancer ${ }^{25}$. 
Although a number of studies have demonstrated that NLR, PLR, MLR and D-Dimer can be used as potential prognostic biomarkers in patients with a variety of solid tumors, these studies have more or less analyzed only one or two of these inflammatory indicators as markers. MLR and D-dimer were also proposed in our study as independent predictive markers of prognosis in patients with surgically treated lung cancer. However, we are not aware of any studies that have examined whether there is superposition between these markers or whether there is an interaction between them. For this reason, we divided these markers into three groups according to the number of beneficial factors, and the results of a multifactorial COX regression show that patients in the group without a single beneficial factor had the worst prognosis compared to the other two groups, while patients in the group with the most beneficial factors had the best prognosis. It is reasonable to believe that the number of beneficial peripheral blood biomarkers described above could be a valid predictor of the prognostic status of patients. There are some shortcomings in our study, as a retrospective study, selection bias may occur in the investigation and therefore the results need to be further clarified in a multicenter prospective study. In addition, only patients with operable early-stage lung cancer were selected, while it is unknown whether patients with inoperable advanced lung cancer have a superimposed effect in these tumor markers, and further studies are needed.

\section{Conclusion}

MLR and D-Dimer can be inexpensive and convenient independent predictors of prognosis in patients with surgically treatable early-stage lung cancer, and their predictive superposition can play a greater role in individualizing patient treatment.

\section{Abbreviations}

MLR: monocyte-to-lymphocyte ratio

NLR: neutrophil-to-lymphocyte ratio

PLR: Platura-to-lymphocyte ratio

D-dimer: dimeric fibrin fragment $D$

LC: Lung Cancer

PFS: Progression-free survival

OS: overall survival

TNM: tumor node metastases

NSCLC: non-small-cell lung cancer 
ROC: receiver operating Characteristic

Cl: confidence interval

HR: risk ratio

CTL: cytotoxic T lymphocytes

\section{Declarations}

\section{Ethics approval and consent to participate}

For this study, the IRB approved our application for waiver of informed consent.Because of our IRB

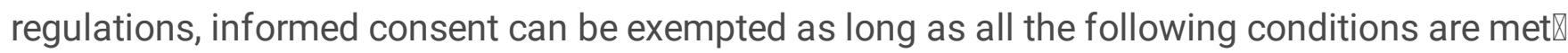

1. The risk to the subjects in this study is not greater than the minimum risk. (Minimum risk refers to the possibility and degree of the expected risk in the research is not greater than the risk of daily life, or routine physical examination or psychological test.)

2. Exemption of informed consent will not adversely affect the rights and health of subjects. Note: The patient only needs to accept the normal diagnosis and treatment process of the disease, and any medical treatment and rights will not be affected.

3. The subjects' privacy and personally identifiable information are protected.Note: The personal information of patients in the study is confidential. Information that can identify patients will not be disclosed to members other than the research team. All study members and study sponsors are required to keep patient identities confidential. The patient file will only be available to researchers. In order to ensure that the research is carried out in accordance with regulations, when necessary, the government management department or the members of the ethics committee can review the patient data in the research unit according to the regulations. When the results of this research are published, no personal information about the patients will be disclosed.

4. If informed consent is required, the research will not be possible (patients have the right to know that their medical records/specimen may be used for research, and their refusal or disagreement to participate in the research is not the reason why the research cannot be implemented or the informed consent is exempted).Note: Patients have the right to know that their tissue samples may be used for research and have the right to refuse to participate in the research.

5. This study does not use medical records and specimens that patients/subjects have specifically refused to use in the past.

6. Research using human body materials or data with identifiable information has failed to find the subject, and the research project does not involve personal privacy or commercial interests.

We believe that our research meets the above conditions. 
This study has been reviewed by the Ethics Committee of the Second Affiliated Hospital of Harbin Medical University, with approval number:KY2021-254.

\section{Consent for publication}

This study is a retrospective study, and the ethics committee's exemption consent application has been passed, and the relevant data is only used in this study

\section{Availability of data and materials}

The datasets generated and/or analysed during the current study are not publicly available due [Our ethics committee stipulates that the information that can identify patients will not be disclosed to members other than the research team] but are available from the corresponding author on reasonable request.Competing interests

The authors declare that they have no competing interests

\section{Funding}

No funding

\section{Authors' contributions}

WJ and LHW wrote the main manuscript text .

XR prepared figures 1.

LT prepared figures 2.

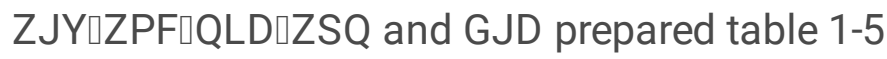

All authors reviewed the manuscript.

\section{Acknowledgements}

In the process of writing the thesis, I also received valuable opinions from many students, and I would like to express my sincere thanks to them. Especially, I wish to thank My best friend Ms. Li Mei for revising my English grammar and suggesting some wording.

\section{References}

(1) Chen, W.; Zheng, R.; Baade, P. D.; Zhang, S.; Zeng, H.; Bray, F.; Jemal, A.; Yu, X. Q.; He, J. Cancer Statistics in China, 2015. CA Cancer J Clin 2016, 66 (2), 115-132. https://doi.org/10.3322/caac.21338.

(2) Gao, S.; Li, N.; Wang, S.; Zhang, F.; Wei, W.; Li, N.; Bi, N.; Wang, Z.; He, J. Lung Cancer in People's Republic of China. Journal of Thoracic Oncology 2020, 15 (10), 1567-1576. 
https://doi.org/10.1016/j.jtho.2020.04.028.

(3) Mayne, N. R.; Lin, B. K.; Darling, A. J.; Raman, V.; Patel, D. C.; Liou, D. Z.; D’Amico, T. A.; Yang, C.-F. J. Stereotactic Body Radiotherapy Versus Delayed Surgery for Early-Stage Non-Small-Cell Lung Cancer. Ann Surg 2020, 272 (6), 925-929. https://doi.org/10.1097/SLA.0000000000004363.

(4) A, G.-C.; A, K.; K, S.; L, G.-K. A Single-Institution Retrospective Analysis of the Differences between 7th and 8th Edition of the UICC TNM Staging System in Patients with Advanced Lung Cancer. European review for medical and pharmacological sciences 2020, 24 (16).

https://doi.org/10.26355/eurrev_202008_22636.

(5) D’Andrea, E.; Choudhry, N. K.; Raby, B.; Weinhouse, G. L.; Najafzadeh, M. A Bronchial-Airway GeneExpression Classifier to Improve the Diagnosis of Lung Cancer: Clinical Outcomes and Cost-Effectiveness Analysis. Int J Cancer 2020, 146 (3), 781-790. https://doi.org/10.1002/ijc.32333.

(6) M, M.; Y, H.; M, I.; S, K.; Y, I.; R, S.; T, U.; T, H.; F, M.; K, N.; K, I.; M, S. Neutrophil-to-Lymphocyte Ratio and Histological Type Might Predict Clinical Responses to Eriburin-Based Treatment in Patients with Metastatic Breast Cancer. Breast cancer (Tokyo, Japan) 2020, 27 (4). https://doi.org/10.1007/s12282020-01069-0.

(7) Tiainen, S.; Rilla, K.; Hämäläinen, K.; Oikari, S.; Auvinen, P. The Prognostic and Predictive Role of the Neutrophil-to-Lymphocyte Ratio and the Monocyte-to-Lymphocyte Ratio in Early Breast Cancer, Especially in the HER2+ Subtype. Breast Cancer Res Treat 2021, 185 (1), 63-72. https://doi.org/10.1007/s10549020-05925-7.

(8) Shiina, Y.; Nakajima, T.; Yamamoto, T.; Tanaka, K.; Sakairi, Y.; Wada, H.; Suzuki, H.; Yoshino, I. The DDimer Level Predicts the Postoperative Prognosis in Patients with Non-Small Cell Lung Cancer. PLoS One 2019, 14 (12), e0222050. https://doi.org/10.1371/journal.pone.0222050.

(9) Ma, J.; Kuzman, J.; Ray, A.; Lawson, B. O.; Khong, B.; Xuan, S.; Hahn, A. W.; Khong, H. T. Neutrophil-toLymphocyte Ratio (NLR) as a Predictor for Recurrence in Patients with Stage III Melanoma. Sci Rep 2018, 8 (1), 4044. https://doi.org/10.1038/s41598-018-22425-3.

(10) Pine, J. K.; Morris, E.; Hutchins, G. G.; West, N. P.; Jayne, D. G.; Quirke, P.; Prasad, K. R. Systemic Neutrophil-to-Lymphocyte Ratio in Colorectal Cancer: The Relationship to Patient Survival, Tumour Biology and Local Lymphocytic Response to Tumour. Br J Cancer 2015, 113 (2), 204-211. https://doi.org/10.1038/bjc.2015.87.

(11) Feng, J.-F.; Huang, Y.; Chen, Q.-X. Preoperative Platelet Lymphocyte Ratio (PLR) Is Superior to Neutrophil Lymphocyte Ratio (NLR) as a Predictive Factor in Patients with Esophageal Squamous Cell Carcinoma. World J Surg Onco/ 2014, 12, 58. https://doi.org/10.1186/1477-7819-12-58. 
(12) Topkan, E.; Mertsoylu, H.; Kucuk, A.; Besen, A. A.; Sezer, A.; Sezen, D.; Bolukbasi, Y.; Selek, U.;

Pehlivan, B. Low Systemic Inflammation Response Index Predicts Good Prognosis in Locally Advanced Pancreatic Carcinoma Patients Treated with Concurrent Chemoradiotherapy. Gastroenterol Res Pract 2020, 2020, 5701949. https://doi.org/10.1155/2020/5701949.

(13) Tanizaki, J.; Haratani, K.; Hayashi, H.; Chiba, Y.; Nakamura, Y.; Yonesaka, K.; Kudo, K.; Kaneda, H.; Hasegawa, Y.; Tanaka, K.; Takeda, M.; Ito, A.; Nakagawa, K. Peripheral Blood Biomarkers Associated with Clinical Outcome in Non-Small Cell Lung Cancer Patients Treated with Nivolumab. Journal of Thoracic Oncology 2018, 13 (1), 97-105. https://doi.org/10.1016/j.jtho.2017.10.030.

(14) Galvano, A.; Peri, M.; Guarini, A. A.; Castiglia, M.; Grassadonia, A.; De Tursi, M.; Irtelli, L.; Rizzo, S.; Bertani, A.; Gristina, V.; Barraco, N.; Russo, A.; Natoli, C.; Bazan, V. Analysis of Systemic Inflammatory Biomarkers in Neuroendocrine Carcinomas of the Lung: Prognostic and Predictive Significance of NLR, LDH, ALI, and LIPI Score. Ther Adv Med Oncol 2020, 12, 1758835920942378.

https://doi.org/10.1177/1758835920942378.

(15) Balkwill, F.; Mantovani, A. Inflammation and Cancer: Back to Virchow? Lancet 2001, 357(9255), 539-545. https://doi.org/10.1016/S0140-6736(00)04046-0.

(16) Diakos, C. I.; Charles, K. A.; McMillan, D. C.; Clarke, S. J. Cancer-Related Inflammation and Treatment Effectiveness. Lancet Oncol 2014, 15(11), e493-503. https://doi.org/10.1016/S1470-2045(14)70263-3.

(17) Elinav, E.; Nowarski, R.; Thaiss, C. A.; Hu, B.; Jin, C.; Flavell, R. A. Inflammation-Induced Cancer: Crosstalk between Tumours, Immune Cells and Microorganisms. Nat Rev Cancer 2013, 13 (11), $759-771$. https://doi.org/10.1038/nrc3611.

(18) Greten, F. R.; Grivennikov, S. I. Inflammation and Cancer: Triggers, Mechanisms, and Consequences. Immunity 2019, 51 (1), 27-41. https://doi.org/10.1016/j.immuni.2019.06.025.

(19) Ray-Coquard, I.; Cropet, C.; Van Glabbeke, M.; Sebban, C.; Le Cesne, A.; Judson, I.; Tredan, O.; Verweij, J.; Biron, P.; Labidi, I.; Guastalla, J.-P.; Bachelot, T.; Perol, D.; Chabaud, S.; Hogendoorn, P. C. W.; Cassier, P.; Dufresne, A.; Blay, J.-Y.; European Organization for Research and Treatment of Cancer Soft Tissue and Bone Sarcoma Group. Lymphopenia as a Prognostic Factor for Overall Survival in Advanced Carcinomas, Sarcomas, and Lymphomas. Cancer Res 2009, 69 (13), 5383-5391. https://doi.org/10.1158/00085472.CAN-08-3845.

(20) Farhood, B.; Najafi, M.; Mortezaee, K. CD8+ Cytotoxic T Lymphocytes in Cancer Immunotherapy: A Review. J Cell Physiol 2019, 234 (6), 8509-8521. https://doi.org/10.1002/jcp.27782.

(21) Ardi, V. C.; Kupriyanova, T. A.; Deryugina, E. I.; Quigley, J. P. Human Neutrophils Uniquely Release TIMP-Free MMP-9 to Provide a Potent Catalytic Stimulator of Angiogenesis. Proc Natl Acad Sci U S A 2007, 104 (51), 20262-20267. https://doi.org/10.1073/pnas.0706438104. 
(22) Coffelt, S. B.; Wellenstein, M. D.; de Visser, K. E. Neutrophils in Cancer: Neutral No More. Nat Rev Cancer 2016, 16 (7), 431-446. https://doi.org/10.1038/nrc.2016.52.

(23) Chittezhath, M.; Dhillon, M. K.; Lim, J. Y.; Laoui, D.; Shalova, I. N.; Teo, Y. L.; Chen, J.; Kamaraj, R.; Raman, L.; Lum, J.; Thamboo, T. P.; Chiong, E.; Zolezzi, F.; Yang, H.; Van Ginderachter, J. A.; Poidinger, M.; Wong, A. S. C.; Biswas, S. K. Molecular Profiling Reveals a Tumor-Promoting Phenotype of Monocytes and Macrophages in Human Cancer Progression. Immunity 2014, 41 (5), 815-829.

https://doi.org/10.1016/j.immuni.2014.09.014.

(24) Schlesinger, M. Role of Platelets and Platelet Receptors in Cancer Metastasis. J Hematol Oncol 2018, 11 (1), 125. https://doi.org/10.1186/s13045-018-0669-2.

(25) Lu, Y.; Zhang, L.; Zhang, Q.; Zhang, Y.; Chen, D.; Lou, J.; Jiang, J.; Ren, C. The Association of D-Dimer with Clinicopathological Features of Breast Cancer and Its Usefulness in Differential Diagnosis: A Systematic Review and Meta-Analysis. PLoS One2019, 14 (9), e0221374.

https://doi.org/10.1371/journal.pone.0221374.

\section{Tables}

Tab 1. Patients' characteristics at baseline 
Characteristic

Age

Sex

Male $\quad 218 \varangle 58 \% \rrbracket$

BMI

Address

Country

Town

Smoking status

\begin{tabular}{|c|c|c|}
\hline & Never & $43.6 \otimes 56.4 \% \rrbracket$ \\
\hline \multirow[t]{5}{*}{ Tumor site } & RUL & $81 \bowtie 21.5 \% \rrbracket$ \\
\hline & LUL & $103 \rrbracket 27.4 \% \rrbracket$ \\
\hline & RLL & 82 『21.8\%】 \\
\hline & LLL & $83 \llbracket 22.1 \% \rrbracket$ \\
\hline & $\mathrm{RML}$ & $27 \otimes 7.2 \% \rrbracket$ \\
\hline
\end{tabular}

Histologic subtype Adeno. $\quad 233 \llbracket 62 \% \rrbracket$

Squamous. $\quad 90 \rrbracket 23.9 \% \rrbracket$

SCLC

$25 \otimes 6.6 \% \bigotimes$

Another

$28 \otimes 7.4 \% \bigotimes$

Differentiation

Well

Moderately $\quad 90 \varangle 23.9 \% \rrbracket$

Poorly $220 \otimes 58.5 \% \bigotimes$

\begin{tabular}{|c|c|c|}
\hline \multirow[t]{2}{*}{ PIS } & Yes & $94 \varangle 25 \% \rrbracket$ \\
\hline & No & $282 \varangle 75 \% \rrbracket$ \\
\hline \multirow[t]{2}{*}{ TNM staging } & I & $205 \rrbracket 54.5 \% \rrbracket$ \\
\hline & II & $171 \rrbracket 45.5 \% \rrbracket$ \\
\hline \multirow[t]{2}{*}{ ECOG PS } & $0-1$ & $320 \rrbracket 85.1 \% \rrbracket$ \\
\hline & $2-4$ & $56 \rrbracket 14.9 \% \rrbracket$ \\
\hline \multirow[t]{2}{*}{ Basic illness } & Hypertension & $59 \rrbracket 15.7 \% \rrbracket$ \\
\hline & Diabetes & $20 \varangle 5.3 \% \rrbracket$ \\
\hline
\end{tabular}

Tumor Size
$60 \rrbracket 53 \llbracket 65 \rrbracket$

$23.86 \pm 3.2$ 

Lung function
FEV1
$2.37 \pm 0.63$
FEV $1 \%$
$78.65 \pm 9.84$
DLCO
$6.74 \pm 1.91$

BMI, body mass index; ECOG PS, Eastern Cooperative Oncology Group performance status; TNM staging,

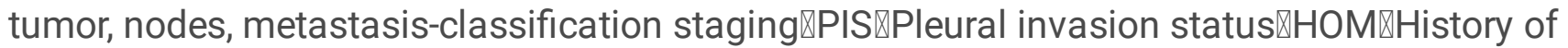

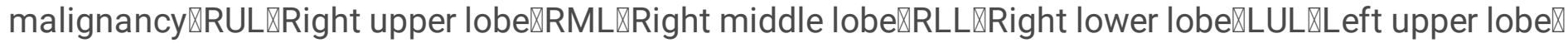
LLL囚Left lower lobe囚

\section{Table 2. Patient's peripheral blood data}

\begin{tabular}{|c|c|c|c|}
\hline \multirow[t]{2}{*}{ Characteristic } & \multirow[t]{2}{*}{ Median $(25 \%, 75 \%)$} & \multicolumn{2}{|c|}{ Total $(n=376)$} \\
\hline & & High $\varangle \otimes$ & low $₫ \% \rrbracket$ \\
\hline Neutrophil count & $4.07 \rrbracket 3.13 \rrbracket 5.04 \llbracket\left(10^{\wedge} 9 / L\right)$ & & \\
\hline Lymphocyte count & $1.87 \rrbracket 1.5 \rrbracket 2.34 \rrbracket\left(10^{\wedge} 9 / L\right)$ & & \\
\hline Monocyte count & $0.36 \rrbracket 0.25 \rrbracket 0.46 \rrbracket\left(10^{\wedge} 9 / L\right)$ & & \\
\hline Platelet count & $237 \rrbracket 196 \rrbracket 285 \rrbracket\left(10^{\wedge} 9 / L\right)$ & & \\
\hline D-Dimer & $110.5 \rrbracket 62.25 \llbracket 168 \rrbracket(\mathrm{ng} / \mathrm{ml})$ & $263 \llbracket 69.7 \rrbracket$ & $113 \varangle 30.3 \rrbracket$ \\
\hline MLR & $0.22 \varangle 0.14 \llbracket 0.25 \rrbracket$ & $137 \rrbracket 36.4 \rrbracket$ & $239 \llbracket 63.6 \rrbracket$ \\
\hline NLR & $2.61 \rrbracket 1.58 \rrbracket 2.94 \rrbracket$ & $207 \rrbracket 55.1 \rrbracket$ & $169 \rrbracket 44.9 \rrbracket$ \\
\hline PLR & $137.6 \rrbracket 96.73 \rrbracket 162.73 \rrbracket$ & $167 \rrbracket 44.4 \rrbracket$ & $209 \llbracket 55.6 \rrbracket$ \\
\hline
\end{tabular}

MLR, monocyte to lymphocyte ratio; NLR, neutrophil to lymphocyte ratio; PLR, platelet to lymphocyte ratio Table3. Univariate analyses of biomarkers for OS and PFS. 


\begin{tabular}{|c|c|c|c|c|c|c|c|}
\hline \multirow[t]{2}{*}{ Characteristic } & \multirow[t]{2}{*}{ Reference } & \multicolumn{3}{|l|}{ OS } & \multicolumn{3}{|l|}{ PFS } \\
\hline & & $\mathrm{HR}$ & $95 \% \mathrm{Cl}$ & p-value & $\mathrm{HR}$ & $95 \% \mathrm{Cl}$ & $\mathrm{p}$-value \\
\hline sex & female & 2.44 & $1.59-3.75$ & 0.000048 & 2.26 & $1.51-3.40$ & 0.000081 \\
\hline age & & 1.01 & $0.99-1.03$ & 0.241034 & 1.01 & $0.99-1.03$ & 0.257189 \\
\hline smoking & no & 1.69 & $1.16-2.46$ & 0.006738 & 1.53 & $1.07-2.20$ & 0.021356 \\
\hline pathology & adeno. & & & & & & \\
\hline another & & 1.73 & $0.85-3.51$ & 0.128456 & 1.70 & $0.87-3.32$ & 0.121719 \\
\hline SCLC & & 2.86 & $1.49-5.47$ & 0.001567 & 2.53 & $1.33-4.81$ & 0.004787 \\
\hline squama. & & 1.96 & $1.29-3.00$ & 0.001793 & 1.82 & $1.21-2.75$ & 0.004036 \\
\hline size & & 1.01 & $0.99-1.02$ & 0.326866 & 1.01 & $1.00-1.02$ & 0.262015 \\
\hline PIS & no & 1.78 & $1.20-2.64$ & 0.003969 & 1.96 & $1.35-2.85$ & 0.000426 \\
\hline TNM & 0 & 1.25 & $0.86-1.83$ & 0.238301 & 1.32 & $0.92-1.89$ & 0.133186 \\
\hline BMI & & 0.97 & $0.92-1.03$ & 0.369197 & 0.98 & $0.93-1.04$ & 0.527315 \\
\hline MLR & high & 0.39 & $0.27-0.57$ & 0.000001 & 0.43 & $0.30-0.61$ & $<0.0001$ \\
\hline NLR & high & 0.46 & $0.31-0.70$ & 0.00021 & 0.49 & $0.33-0.72$ & 0.000252 \\
\hline PLR & high & 0.57 & $0.39-0.83$ & 0.003706 & 0.64 & $0.44-0.91$ & 0.014286 \\
\hline D-Dimer & high & 0.40 & $0.24-0.67$ & 0.000453 & 0.57 & $0.37-0.89$ & 0.012654 \\
\hline ECGO PS & $2-4$ & 0.26 & $0.18-0.39$ & $<0.0001$ & 0.21 & $0.14-0.31$ & $<0.0001$ \\
\hline
\end{tabular}

Table4. multivariate analyses of biomarkers for OS and PFS. 


\begin{tabular}{|llllllll|}
\hline Characteristic & Reference & OS & \multicolumn{5}{c|}{ PFS } \\
\cline { 3 - 8 } & & HR & $95 \% \mathrm{Cl}$ & p-value & HR & $95 \% \mathrm{Cl}$ & p-value \\
\hline sex & female & 1.72 & $1.06-2.80$ & 0.02843 & 1.51 & $0.96-2.39$ & 0.077308 \\
\hline $\begin{array}{l}\text { Pathology } \\
\text { another }\end{array}$ & adeno. & & & & & & \\
SCLC & & 1.41 & $0.69-2.90$ & 0.344902 & 1.47 & $0.75-2.91$ & 0.265219 \\
squama. & & 2.25 & $1.14-4.44$ & 0.018932 & 2.44 & $1.26-4.72$ & 0.008322 \\
\hline PIS & no & 1.56 & $0.98-2.46$ & 0.05821 & 1.61 & $1.03-2.51$ & 0.037925 \\
\hline MLR & high & 0.56 & $0.37-0.87$ & 0.009145 & 0.57 & $0.39-0.85$ & 0.005487 \\
\hline PLR & high & 0.74 & $0.49-1.12$ & 0.157511 & & & \\
\hline D-Dimer & high & 0.48 & $0.29-0.80$ & 0.005376 & 0.67 & $0.43-1.04$ & 0.076619 \\
\hline ECGO PS & $2-4$ & 0.28 & $0.18-0.42$ & $<0.0001$ & 0.23 & $0.15-0.34$ & $<0.0001$ \\
\hline
\end{tabular}

Table5. Multivariate COX regression analysis of OS and PFS grouped by beneficial factors

\begin{tabular}{|c|c|c|c|c|c|c|c|}
\hline \multirow[t]{2}{*}{ group } & \multirow[t]{2}{*}{ Total囚n=376凹 } & \multicolumn{3}{|l|}{ os } & \multicolumn{3}{|l|}{ PFS } \\
\hline & & $\mathrm{HR}$ & $95 \% \mathrm{Cl}$ & p-value & $\mathrm{HR}$ & $95 \% \mathrm{Cl}$ & p-value \\
\hline$\otimes(0)$ & 68 & \multicolumn{3}{|c|}{ Reference } & \multicolumn{3}{|c|}{ Reference } \\
\hline$\square(1-2)$ & 164 & 0.40 & $0.26-0.62$ & $<0.0001$ & 0.48 & $0.31-0.74$ & 0.00083 \\
\hline$\bigotimes(3-4)$ & 144 & 0.29 & $0.17-0.48$ & $<0.0001$ & 0.37 & $0.23-0.59$ & $<0.0001$ \\
\hline
\end{tabular}

Covariables included age, Eastern Cooperative Oncology Group performance status (0-1 or 2-4), smoking

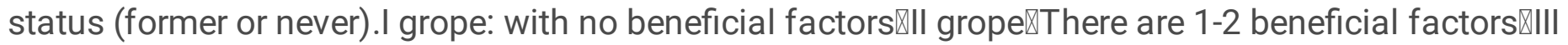
grope囚There are 3-4 beneficial factors

\section{Figures}


A

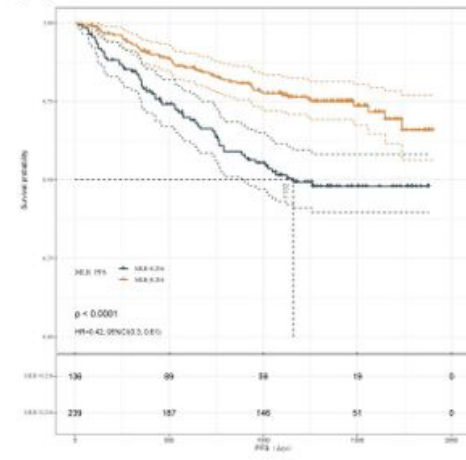

E

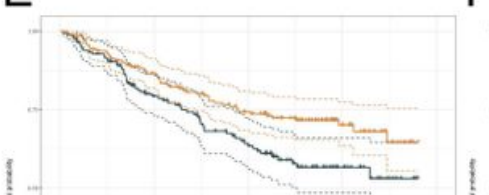

B

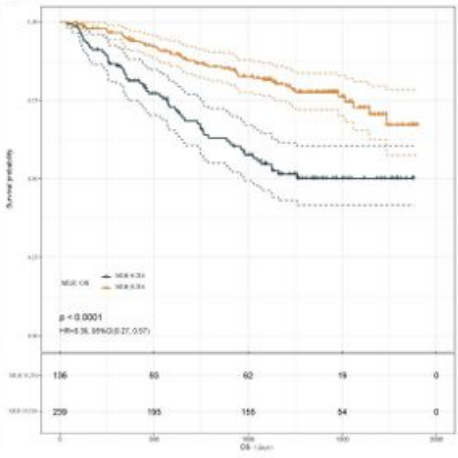

$\mathrm{F}$

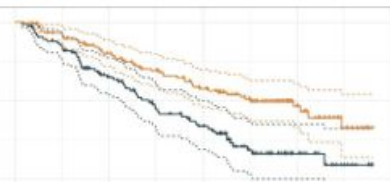

C

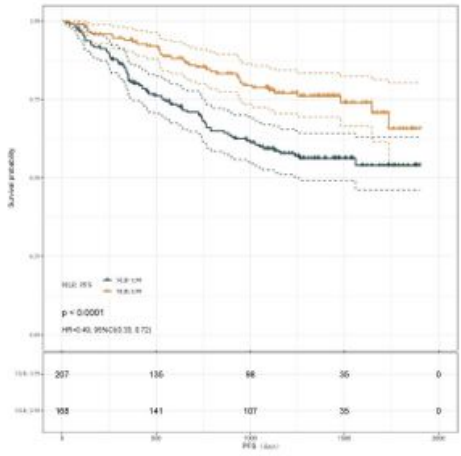

G

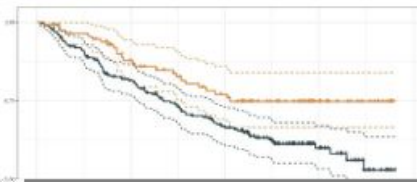

D

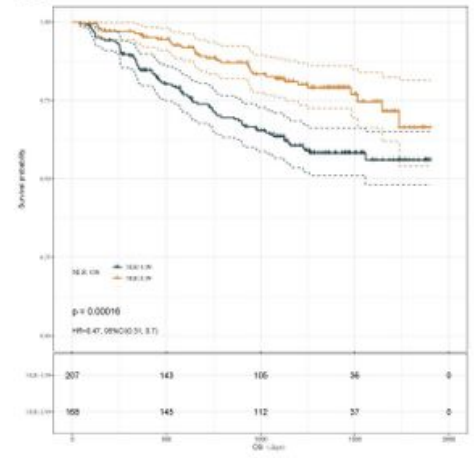

$\mathrm{H}$

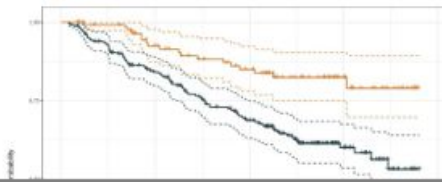

Figure 1

PFS $(a, c, e, g)$ and OS $(b, d, f, h)$ curves of patients stratified according to peripheral blood markers (MLR, NLR,PLR and D-dimer). $p$ Values were calculated with the log-rank test

A

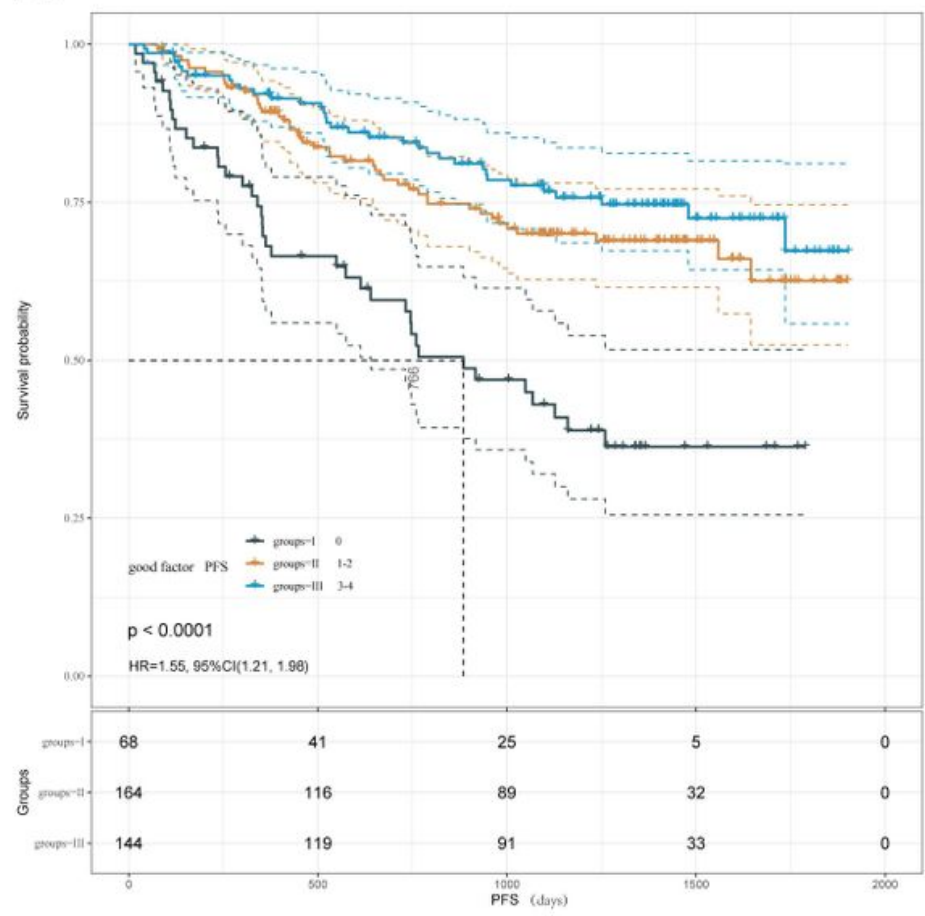

B

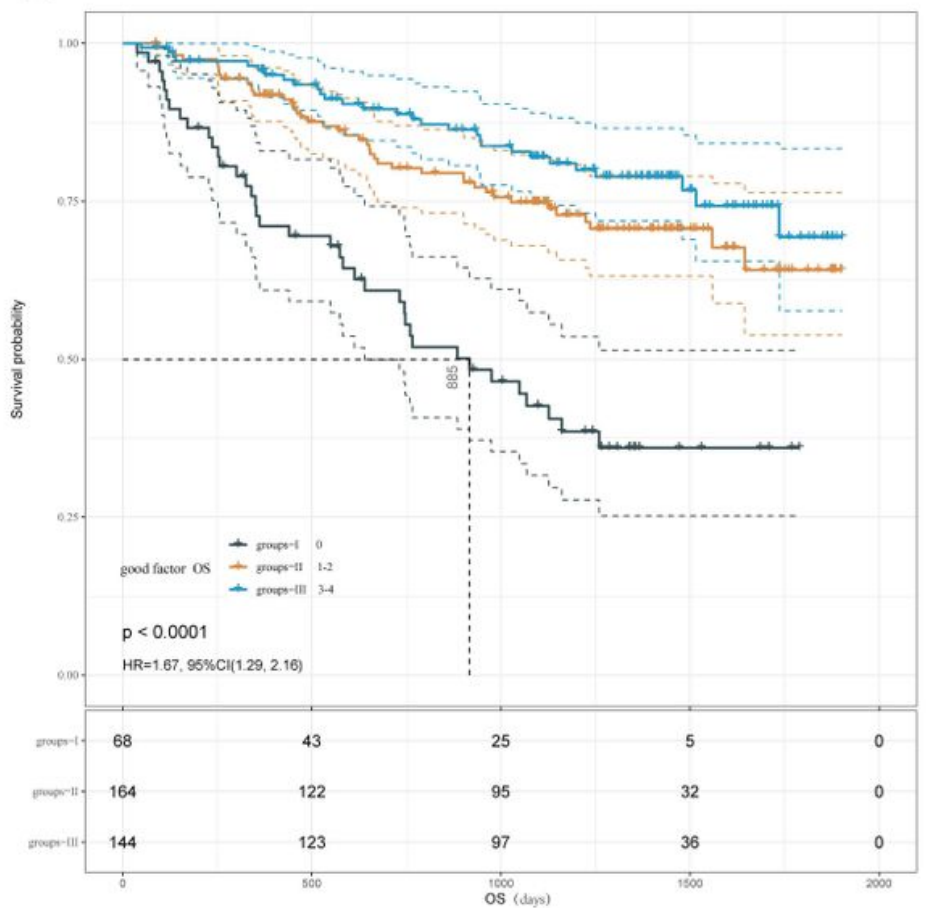


Figure 2

PFS rate (A), and OS rate (B) were determined for patients in groups I, II, and III (non one,one and two ,three and four factors, respectively). $p$ Values were calculated with the log-rank test 